\title{
Mutational Analysis of the Metal-binding Sites of Peroxide Sensor PerR
}

\author{
Young-Bin Won, Chang-Jun Ji, Ju Hyun Cho, ${ }^{\dagger, *}$ and Jin-Won Lee* \\ Department of Life Science and Research Center for Natural Sciences, Hanyang University, Seoul 133-791, Korea \\ "E-mail: jwl@hanyang.ac.kr \\ 'Department of Biology, Research Institute of Life Science, Gyeongsang National University, Jinju 660-701, Korea \\ ${ }^{*}$ E-mail: juhyun.cho@gnu.ac.kr \\ Received April 8, 2010, Accepted April 27, 2010
}

\begin{abstract}
Bacillus subtilis PerR is a metal-dependent peroxide-sensing transcription factor which uses metal-catalyzed histidine oxidation for peroxide-sensing. PerR contains two metal binding sites, one for structural $\mathrm{Zn}^{2+}$ and the other for the regulatory/peroxide-sensing metal. Here we investigated the effect of mutations at both the structural and regulatory metal binding sites on the oxidation of either $\mathrm{H} 37$ or H91, two of the peroxide-sensing ligands. All four serine substitution mutants at the structural $\mathrm{Zn}^{2+}$ site (C96S, C99S, C136S and C139S) exhibited no detectable oxidation at histidine residues. Two of the alanine substitution mutants at regulatory metal site (H37A and D85A) exhibited selective oxidation preferentially at the H91-containing tryptic peptide, whereas no oxidation was detected in the other mutants (H91A, H93A and D104A). Our results suggest that the cysteine residues coordinating structural $\mathrm{Zn}^{2+}$ are essential for peroxide sensing by PerR, and that the C-terminal regulatory metal binding site composed of H91, H93 and D104 can bind Fe ${ }^{2+}$, providing a possible explanation for the peroxide sensing mechanisms by PerR.
\end{abstract}

Key Words: PerR, Bacillus subtilis, Peroxide, Metal, Transcription factor

\section{Introduction}

Reactive oxygen species (ROS) such as superoxide $\left(\mathrm{O}_{2}{ }^{-}\right)$, hydrogen peroxide $\left(\mathrm{H}_{2} \mathrm{O}_{2}\right)$ and hydroxyl radical $(\mathrm{HO} \bullet)$ are generated as inadvertent by-products of aerobic metabolism. In addition to this, large doses of ROS are actively generated by plants and animals, and even by microbes, that wish to suppress the growth of bacteria. ${ }^{1}$ Since ROS can damage proteins, lipids and nucleic acids leading to mutagenesis or cell death, bacteria possess defense systems against oxidative stress caused by ROS. The defense systems are typically coordinated by transcription factors that sense ROS and regulate genes involved in detoxification of ROS. ${ }^{1}$

One of the best-characterized bacterial sensors of ROS is Escherichia coli OxyR transcriptional factor. Like other peroxide sensors such as RsrA, Hsp33, OhrR, yeast Orp1-Yap1 and eukaryotic protein tyrosine phosphastases, OxyR uses redoxactive cysteine residue for the sensing of peroxide stress. ${ }^{2-7}$ However, Bacillus subtilis, a model Gram positive soil bacterium, lacks the OxyR and instead it has PerR as the major regulator of the inducible hydrogen peroxide stress response. ${ }^{8-9}$ PerR controls genes involved in detoxification of peroxides and in minimization of the damage from intracelluar Fe-catalyzed redox reactions, making it functionally analogous to $E$. coli OxyR. PerR regulon includes genes coding for a Dps-like DNAbinding protein MrgA, the major vegetative catalase KatA, alkyl hydroperoxidase AhpCF, heme biosynthesis enzymes HemAXCDBL, a $\mathrm{Zn}^{2+}$ uptake ATPase ZosA, iron uptake regulator Fur and PerR itself.

PerR is a homodimeric protein and contains two metal binding sites in each monomer: a structural $\mathrm{Zn}^{2+}$ binding site coordinated by four cysteines (Cys4:Zn site) and a regulatory metal binding site which can be occupied by either iron (PerR:Zn,Fe)
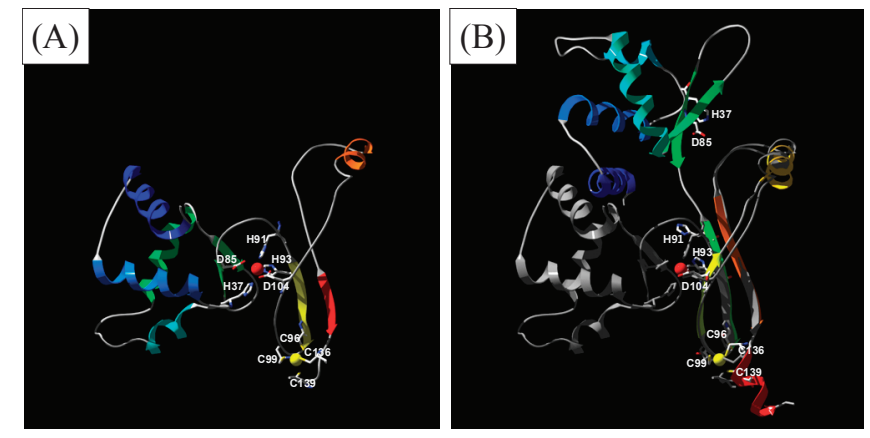

Figure 1. Structure of PerR protein. (A) Structure of active PerR:Zn,Mn (PDB accession number of $3 \mathrm{~F} 8 \mathrm{~N}$ ). The structural $\mathrm{Zn}^{2+}$ ion shown in yellow is coordinated by four cysteine residues (C96, C99, C136 and C139) and the regulatory $\mathrm{Mn}^{2+}$ shown in red is coordinated by H37, D85, H91, H93 and D104. (B) Structure of apo-PerR (PerR:Zn) (PDB accession number of 2FE3) superimposed with active PerR:Zn,Mn (gray). The structural $\mathrm{Zn}^{2+}$ ion is still coordinated by four cysteine residues as in active PerR:Zn,Mn, however the regulatory metal binding site is separated into two parts, the N-terminal domain containing H37 and D85 and C-terminal domain containing H91, H93 and D104.

or manganese (PerR:Zn,Mn) (Fig. 1A). ${ }^{10-13}$ Although both the metallated forms of PerR (PerR:Zn,Fe and PerR:Zn,Mn) can act as active repressors, these two forms of PerR show dramatic difference in their response to hydrogen peroxide. In contrast to PerR:Zn,Mn which is poor peroxide sensor, PerR:Zn,Fe can sense physiologically relevant (micromolar) levels of peroxide rapidly. Recent biochemical and structural studies have established that PerR uses totally different mechanisms for the sensing of low levels of peroxide in contrast to other peroxide sensors that use redox active cysteines for the sensing of peroxide. ${ }^{13-14}$ The reaction of PerR: $\mathrm{Zn}$, Fe with $\mathrm{H}_{2} \mathrm{O}_{2}$ leads to the Fe-catalyzed oxidation of either $\mathrm{H} 37$ or $\mathrm{H} 91$, two of the ligands for the re- 
gulatory metal ion.

Each monomer of PerR is composed of two domains, N-terminal DNA binding domain (residues 2 - 85) and C-terminal dimerization domain (residues 92 - 144) connected by a loop (residues 86 - 91) (Fig. 1A). ${ }^{15}$ In the absence of regulatory metal ion, the apo-PerR (PerR:Zn) adopts a rather flat conformation, where the two DNA-binding domains project opposite directions from the dimerization domain (Fig. 1B). The regulatory metal binding brings the $\mathrm{N}$ - and $\mathrm{C}$-terminal domains together leading to a perpendicular caliper-like conformation more suited for DNA binding (Fig. 1A).

Previously we showed that PerR proteins with mutations at the structural $\mathrm{Zn}^{2+}$ binding site cannot bind $\mathrm{Zn}^{2+}$ and thus are structurally unstable, and showed that Ala substitution mutants of regulatory metal binding site still bind $\mathrm{Zn}^{2+}$, but no longer act as functional repressors in vivo. ${ }^{13}$ However, there is no report on the oxidation of PerR with mutations at the structural $\mathrm{Zn}^{2+}$ site. Furthermore, the oxidation of two peroxide-sensing histidine residues, $\mathrm{H} 37$ and $\mathrm{H} 91$, was not systematically investigated for the mutant proteins. In the present study, we overexpressed all the structural $\mathrm{Zn}^{2+}$ - and the regulatory metal-binding site mutant proteins in $E$. coli and investigated the oxidation of these proteins after $\mathrm{H}_{2} \mathrm{O}_{2}$ treatment using MALDI-TOF MS.

\section{Materials and Methods}

Construction of PerR variant overexpressing $E$. coli strains. The $E$. coli strains overexpressing PerR variant proteins were constructed as previously described. ${ }^{10,13}$ Briefly the wild type perR gene open reading frame (ORF) was PCR-amplified using a primer pair of JL69 (5'- GGT GCA TGA CCC ATG GCT GCA CAT-3') and JL70 (5'-TGA CCG TTT CGT GGG ATC CGC TTA-3') with B. subtilis CU1065 genomic DNA as template (bases corresponding to $\mathrm{NcoI}$ and $\mathrm{BamHI}$ sites are underlined). The mutant perR ORFs were amplified using a same primer pair with pDG1730 plasmids harboring mutant perR genes that were generated by Quick-Change site-directed mutagenesis protocol (Stratagene). The PCR fragments were cloned into the NcoI and BamHI sites of overexpression vector pET16b (Novagen) resulting in plasmids named pJL041 (pET16b::perR), pJL042 (pET16b::perR-C96S), pJL043 (pET16b::perR-C99S), pJL044 (pET16b::perR-C136S), pJL047 (pET16b::perR-C139S), pJL 051 (pET16b::perR-H37A), pJL052 (pET16b::perR-D85A), pJL053 (pET16b::perR-H93A), pJL054 (pET16b::perR-D104A) and pJL058 (pET16b::perR-H91A). These plasmids were introduced into E. coli BL21 (DE3) pLysS resulting in PerR variant overexpressing E. coli strains.

MALDI-TOF mass analysis. Each $E$. coli strain harboring perR variant gene was grown in $5 \mathrm{~mL}$ of LB medium containing $0.4 \%$ glucose, $50 \mu \mathrm{g} / \mathrm{mL}$ ampicillin and $34 \mu \mathrm{g} / \mathrm{mL}$ chlorampenicol at $37^{\circ} \mathrm{C}$. At $\mathrm{OD}_{600} \sim 0.4$, cells were treated with $1 \mathrm{mM}$ Isopropyl 1-thio- $\beta$-D-galactopyranoside and allowed to grow for an additional $2 \mathrm{~h}$ to induce PerR variant expression. Aliquots of $1.8 \mathrm{~mL}$ of cell culture were untreated or treated with $1 \mathrm{mM} \mathrm{H}_{2} \mathrm{O}_{2}$ (also with $100 \mu \mathrm{M} \mathrm{H}_{2} \mathrm{O}_{2}$ for $E$. coli cells expressing wild type PerR) for $1 \mathrm{~min}$. Cells were harvested by centrifugation after the addition of $200 \mu \mathrm{L}$ of $100 \%$ trichloroacetic acid (TCA). Then, cells were resuspended in $500 \mu \mathrm{L}$ of $10 \%$ TCA and sonicated.
After harvesting sonicated samples by centrifugation for $5 \mathrm{~min}$ at $12000 \times \mathrm{g}$, the pellets were resuspended with $20 \mu \mathrm{L}$ IA buffer (50 mM iodoacetamide, $0.5 \mathrm{M}$ Tris pH 8.0, 5\% glycerol, $100 \mathrm{mM}$ $\mathrm{NaCl}, 1 \mathrm{mM}$ EDTA, 2\% SDS) and incubated for $1 \mathrm{~h}$ in the dark at $25{ }^{\circ} \mathrm{C}$ to alkylate free thiols. Protein samples of $10 \mu \mathrm{L}$ were subjected to $13.3 \%$ Tris-Tricine SDS-PAGE, and protein bands corresponding to PerR variants were cut after staining with Coomassie Brilliant Blue R and subsequent destaining. The gel pieces were washed two times with $100 \mu \mathrm{L}$ of $40 \%$ acetonitrile and $200 \mathrm{mM}$ ammonium bicarbonate for $15 \mathrm{~min}$ at $42{ }^{\circ} \mathrm{C}$, and dried for $45 \mathrm{~min}$ in a SpeedVac. The dried gel pieces were rehydrated with $10 \mu \mathrm{L}$ of trypsin solution ( $20 \mathrm{ng} / \mu \mathrm{L}$ trypsin in $40 \mathrm{mM}$ ammonium bicarbonate containing $9 \%$ acetonitrile). The samples were digested overnight after addition of $10 \mu \mathrm{L}$ of $40 \mathrm{mM}$ ammonium bicarbonate containing $9 \%$ acetonitrile. A total of 0.5 $\mu \mathrm{L}$ of protein digests were mixed with equal volume of matrix solution (saturated solution of $\alpha$-cyano-4-hydroxycinnamic acid, $50 \%$ acetonitrile and $0.1 \%$ TCA) on the target plate and air dried. MALDI-TOF mass spectra were acquired using a Voyager-DE STR instrument (Applied Biosystems) and manipulated using the Data Explorer V4.5 (Applied Biosystems).

\section{Results and Discussion}

Oxidation of wild type PerR in E. coli. PerR is known to be highly susceptible to oxidation during purification. To monitor oxidation of PerR as expressed, E. coli cells overexpressing PerR were precipitated with TCA and free thiol groups were alkylated with iodoacetamide to minimize protein oxidation during sample preparation. Then the total proteins were separated on SDS-PAGE gels without further purification, and the protein bands corresponding to PerR were analyzed by MALDITOF MS after trypsin digestion. As shown in Fig. 2A, the tryptic peptides containing $\mathrm{H} 37$ and $\mathrm{H} 91$ (T5, $\mathrm{m} / \mathrm{z}=2401.2$ and $\mathrm{T} 11^{*}$, $m / z=2199.9$ ) were detected with decreased intensity, and the peptides corresponding to T5 +16 (containing 2-oxo H37) and T11*+16 (containing 2-oxo H91) were detected with increased intensity in samples treated with $100 \mu \mathrm{M} \mathrm{H}_{2} \mathrm{O}_{2}$. The overall oxidation pattern by $\mathrm{H}_{2} \mathrm{O}_{2}$ is comparable to those previously observed with epitope-tagged PerR-FLAG recovered from $B$. subtilis by immunoprecipitation and with Fe-reconstituted PerR after purification. ${ }^{13}$ No significant further oxidation was observed with $1 \mathrm{mM} \mathrm{H}_{2} \mathrm{O}_{2}$ (Fig. 2A) indicating that the incomplete oxidations of $\mathrm{H} 37$-containing peptide and $\mathrm{H} 91$-containing peptide are not caused by insufficient $\mathrm{H}_{2} \mathrm{O}_{2}$ concentrations, and therefore supporting the previous hypothesis that oxidation at one site prevents the oxidation at the other residue. ${ }^{13}$ All these results suggest that the intracelluar metal ion availability to PerR and the intracellular redox status of $E$. coli are very similar to those of $B$. subtilis. Furthermore, our finding that $B$. subtilis PerR is as efficiently oxidized in $E$. coli as in B. subtilis provides a facile method for evaluating PerR oxidation without purification.

Oxidation of $\mathbf{Z n}^{2+}$ binding site mutant proteins. B. subtilis PerR contains a structural $\mathrm{Zn}$ binding site coordinated by four cysteines (Cys4:Zn site) which locks together the three $\beta$-strands in the dimerization domain and stabilizes the dimeric conformation (Fig. 1). ${ }^{10,12-13,15}$ To investigate the role of structural $\mathrm{Zn}^{2+}$ site in peroxide sensing by His oxidation, mutant proteins altered in 

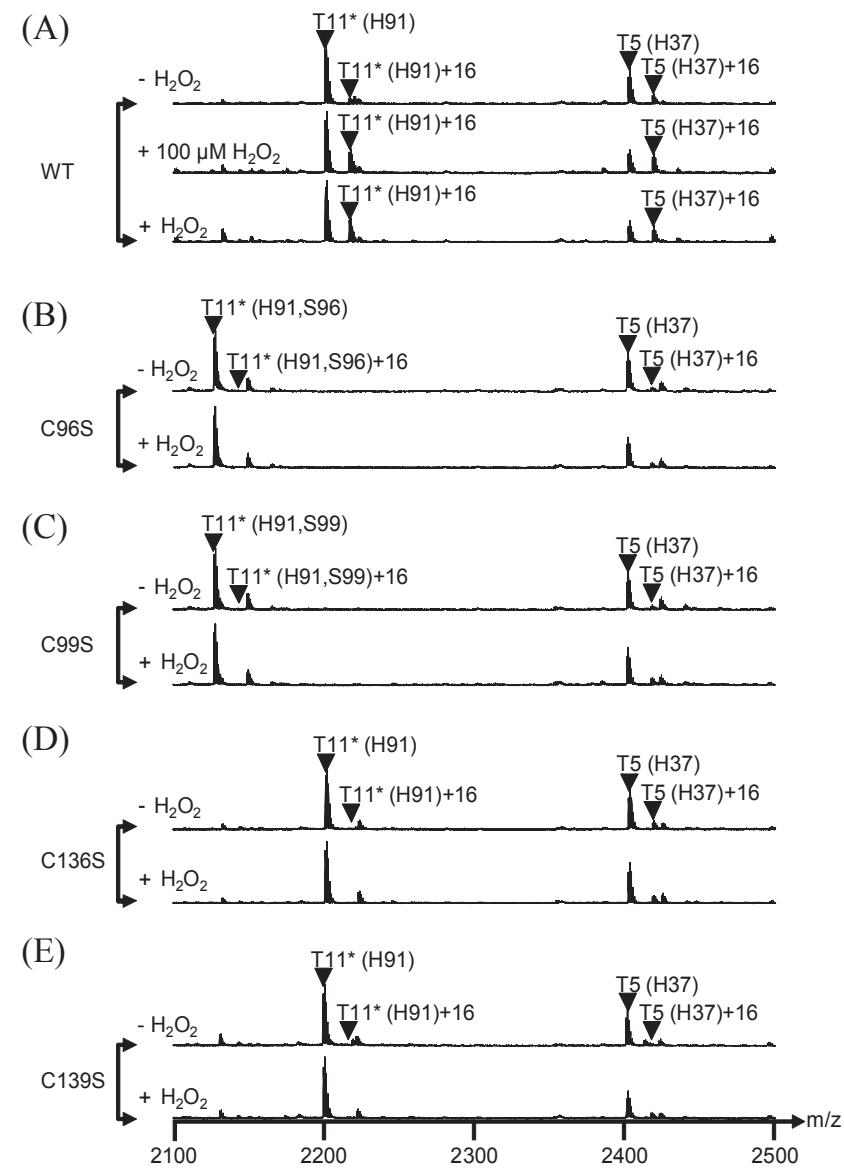

Figure 2. MALDI-TOF analysis of oxidation products for wild type PerR and structural $\mathrm{Zn}^{2+}$ binding site mutants. (A) Wild type PerR overexpressed in $E$. coli was not treated with $\mathrm{H}_{2} \mathrm{O}_{2}$ (denoted as $-\mathrm{H}_{2} \mathrm{O}_{2}$ ), or treated with $100 \mu \mathrm{M} \mathrm{H}_{2} \mathrm{O}_{2}$ or with $1 \mathrm{mM} \mathrm{H}_{2} \mathrm{O}_{2}$ (denoted as $+\mathrm{H}_{2} \mathrm{O}_{2}$ ), and analyzed by MALDI-TOF MS after in-gel tryptic digestion. (B-E) Structural $\mathrm{Zn}^{2+}$ binding site mutant PerR proteins, C96S (B), C99S (C), $\mathrm{C} 136 \mathrm{~S}$ (D) and $\mathrm{C} 139 \mathrm{~S}(\mathrm{E})$, were not treated with $\mathrm{H}_{2} \mathrm{O}_{2}\left(-\mathrm{H}_{2} \mathrm{O}_{2}\right)$ or treated with $1 \mathrm{mM} \mathrm{H}_{2} \mathrm{O}_{2}$ and analyzed by MALDI-TOF MS. Asterisks represent the tryptic peptide containing one (B and C) or two (A, D and E) iodoacetamide-modified carboxyamidomethylated cysteine residues.

any of the four cysteine residues were analyzed by MALDI-TOF MS as described above. All the cysteine to serine substitution mutant proteins (C96S, C99S, C136S, C139S) exhibited no detectable oxidation of either T5 containing H37 or T11 containing H91 (Fig. 2). In addition, the residual cysteine residues, except for the one substituted with serine, were fully modified by iodoacetamide indicating that they were reduced (Supporting information). Previously we have shown that all the cysteine to serine substitution mutants cannot bind $\mathrm{Zn}^{2+}$ and retain no repressor activity in vivo. ${ }^{13}$ These results, together with our previous result, suggest that the structural stability of PerR conferred by Cys $4: \mathrm{Zn}$ site is a prerequisite for the regulatory metal binding and peroxide sensing as well as for the repressor activity.

Oxidation of regulatory metal binding site mutant proteins. The regulatory metal binding site of PerR is composed of five N/O donor ligands: H37, D85, H91, H93 and D104 (Fig. 1A). ${ }^{12-13}$ The alanine substitution mutants of these residues still bind structural $\mathrm{Zn}^{2+}$, however these mutant proteins cannot
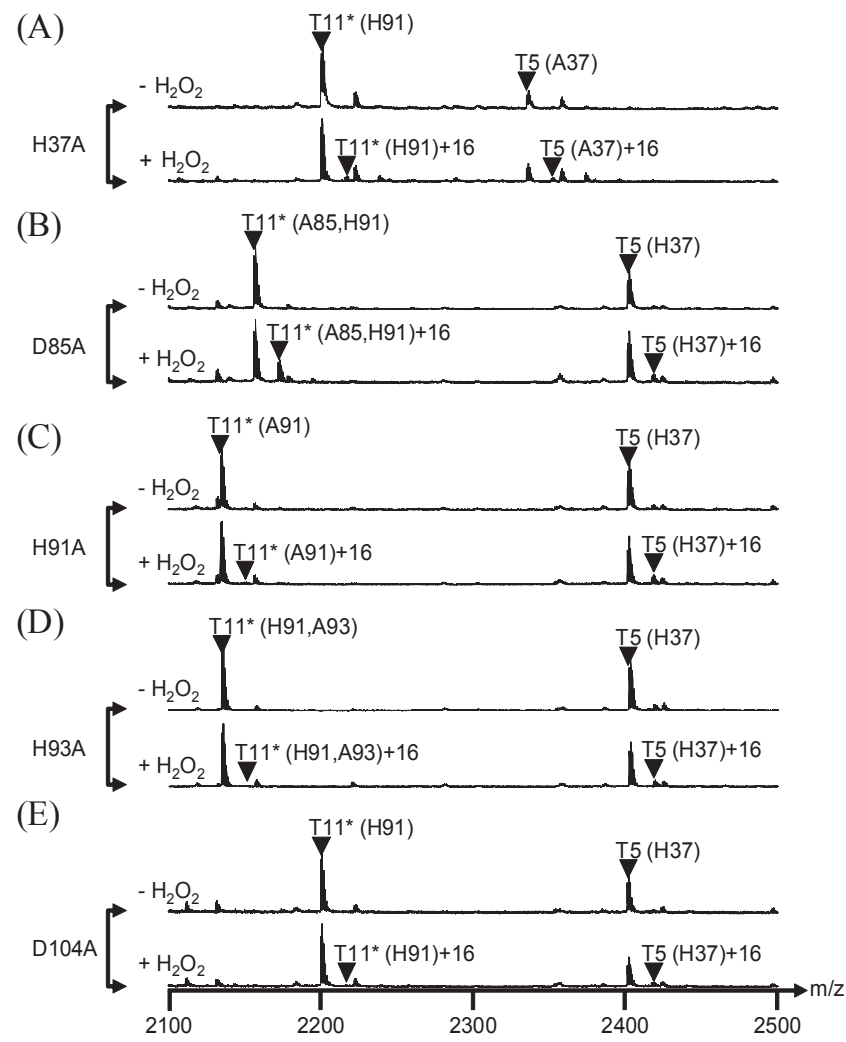

Figure 3. MALD-TOF analysis of oxidation products for regulatory metal binding site mutants. H37A (A), D85A (B), H91A (C), H93A (D) and D104A (E) proteins were overexpressed in E. coli and analyzed by MALDI-TOF MS without $\mathrm{H}_{2} \mathrm{O}_{2}$ treatment $\left(-\mathrm{H}_{2} \mathrm{O}_{2}\right)$ or after $1 \mathrm{mM} \mathrm{H}_{2} \mathrm{O}_{2}$ treatment $\left(+\mathrm{H}_{2} \mathrm{O}_{2}\right)$. Asterisks represent the tryptic peptide containing two iodoacetamide-modified carboxyamidomethylated cysteine residues.

function as active repressors in vivo. In addition, it has been previously shown that alanine substitution of either H37 or H91 eliminates oxidation of $\mathrm{H} 91$ or H37, respectively. ${ }^{13}$ These observations led to the hypothesis that all the five amino acid residues are essential for the regulatory metal binding as well as the repressor activity of PerR. However, it should be noted that the oxidation of histidine residues was investigated using proteins recovered from $B$. subtilis which harbors inactive mutant PerR. In the absence of active PerR the peroxide detoxifying systems including catalase and peroxidase are highly expressed in $B$. subtilis, thus the elimination of histidine oxidation after $\mathrm{H}_{2} \mathrm{O}_{2}$ treatment could simply have resulted from increased peroxide defense systems in B. subtilis. Furthermore the effects of other alanine substitution mutations (D85A, H93A and D104A) on the oxidation of $\mathrm{H} 37$ or $\mathrm{H} 91$ were not investigated in the previous study. $^{13}$

Since the evaluation of PerR oxidation after heterologous expression in $E$. coli was proved to be very efficient as described above, the alanine substitution mutants of regulatory metal binding site were analyzed by MALDI-TOF MS after overexpression in E. coli. As expected H91A mutant protein containing A91 instead of $\mathrm{H} 91$ showed no oxidation of $\mathrm{T} 11 *$ peptide before and after $\mathrm{H}_{2} \mathrm{O}_{2}$ treatment (Fig. 3C). $\mathrm{H} 93$ and D104 mutants that still have intact $\mathrm{H} 91$ also exhibited little, if any, evidence of H91 
oxidation before and after $\mathrm{H}_{2} \mathrm{O}_{2}$ treatment. Unexpectedly, however, D85A mutant showed significantly increased T11*+16 peak intensity indicating oxidation at H91 (Fig. 3B). H37A mutant also showed, though to a lesser extent, increased $\mathrm{T} 11^{*}+16$ peak intensity (Fig. 3A). The slight increase of the peak corresponding to $\mathrm{T} 5+16$, which is consistently observed with all the mutant proteins including H37A mutant lacking H37, is likely due to oxidation of M35 as previously reported (Fig. $3)^{10,13}$ It has been known that the oxidation of H91 (and presumably H37) does not occur in the absence of $\mathrm{Fe}^{2+}$ at the regulatory metal binding site even under severe oxidation conditions. ${ }^{10}$ Thus the oxidation of H91 indicates that D85A and H37A mutants still can bind $\mathrm{Fe}^{2+}$ at the regulatory metal site, although these mutants cannot function as active repressors in vivo. It is likely that the relatively low level of $\mathrm{H} 91$ oxidation in H37A mutant is caused by the observed decrease in metal binding affinity of H37A mutant described previously. ${ }^{16}$ The total absence of histidine oxidation in the H91, H93 and D104 strongly suggest that mutations at the $\mathrm{C}$-terminal metal binding site preclude these mutants binding $\mathrm{Fe}^{2+}$.

It has been previously shown that all the Ala substitution mutants at the regulatory metal binding site cannot function as active repressors. Thus, it is likely that these mutant PerR proteins also adopt a conformation similar to apo-PerR and oxidized PerR which are poorly suited for DNA binding. ${ }^{15-16}$ In this conformation the regulatory metal binding site is separated into two parts, the N-terminal domain containing H37 and D85 and C-terminal domain containing H91, H93 and D104 (Fig. 1B). Although it is not clear whether the $\mathrm{H} 37$ (or D85) is still involved in $\mathrm{Fe}^{2+}$ coordination in $\mathrm{D} 85 \mathrm{~A}$ (or $\mathrm{H} 37 \mathrm{~A}$ ) mutant, it is reasonable to speculate that, based on the structural information, $\mathrm{Fe}^{2+}$ can bind only the C-terminal regulatory metal site which is located away from the $\mathrm{N}$-terminal regulatory metal binding site as shown in Fig. 1B. The ability of PerR to bind $\mathrm{Fe}^{2+}$ in the absence of $\mathrm{H} 37$ and/or D85 can explain our previous observation that multiple oxidation of PerR at both $\mathrm{H} 37$ and H91 can occur by regeneration of $\mathrm{Fe}^{2+}$ under redox recycling conditions. ${ }^{13}$ Furthermore, these results are consistent with the previous observation that H37A mutant and PerR with oxidized $\mathrm{H} 37$ retain $\mathrm{Mn}^{2+}$-binding ability but not H91A mutant and PerR with oxidized H91. ${ }^{16}$ Thus our results strongly support the previous hypothesis that the oxidation of H91 leads to an inactive PerR through loss of metal binding at the regulatory metal binding site. Our results also support the overall idea that the inactivation of PerR by H37 oxidation proceeds through loss of proper coordination by H37. However, in detail, our results suggest that the oxidation of $\mathrm{H} 37$ leads to an inactive $\mathrm{Fe}^{2+}$-bound PerR where the regulatory metal ion is coordinated only by C-terminal metal binding site containing H91, H93 and D104 without the involvement of N- terminal binding site containing D85.

In conclusion we have shown that $B$. subtilis PerR heterologously expressed in $E$. coli system responds to peroxide in a very similar pattern to PerR in B. subtilis, providing an easy and efficient way to monitor PerR oxidation. Using this approach, we have successfully proved that all the cysteine residues coordinating structural $\mathrm{Zn}^{2+}$ are required for the histidine oxidation, suggesting that structural integrity is a prerequisite for the binding of regulatory metal ion and the proper sensing of peroxide by $B$. subtilis PerR. We have further analyzed PerR proteins with mutations at the regulatory metal binding site, and showed that Cterminal regulatory metal binding site can bind $\mathrm{Fe}^{2+}$ and lead to the oxidation of histidine residue, providing a possible explanation for the peroxide sensing mechanisms of metal dependent peroxide sensor, PerR.

Acknowledgments. This work was supported by the research fund of Hanyang University (HY-2007-00000005935).

Suppiementary Information. Extended mass spectra of PerR variants are available on request from the corresponding author (Fax: 82-2-2299-3495, E-mail: jwl@hanyang.ac.kr).

\section{References}

1. Imlay, J. A. Annu. Rev. Biochem. 2008, 77, 755.

2. Paget, M. S.; Buttner, M. J. Annu. Rev. Genet. 2003, 37, 91.

3. Lee, J. W.; Soonsanga, S.; Helmann, J. D. Proc. Natl. Acad. Sci. USA 2007, 104, 8743.

4. Wood, M. J.; Storz, G.; Tjandra, N. Nature 2004, 430, 917.

5. Kang, J. G.; Paget, M. S.; Seok, Y. J.; Hahn, M. Y.; Bae, J. B.; Hahn, J. S.; Kleanthous, C.; Buttner, M. J.; Roe, J. H. EMBO J. 1999, $18,4292$.

6. Ilbert, M.; Horst, J.; Ahrens, S.; Winter, J.; Graf, P. C.; Lilie, H.; Jakob, U. Nat. Struct. Mol. Biol. 2007, 14, 556.

7. van Montfort, R. L.; Congreve, M.; Tisi, D.; Carr, R.; Jhoti, H. Nature 2003, 423, 773.

8. Mongkolsuk, S.; Helmann, J. D. Mol. Microbiol. 2002, 45, 9.

9. Zuber, P. Annu. Rev. Microbiol. 2009, 63, 575.

10. Lee, J. W.; Helmann, J. D. J. Biol. Chem. 2006, 281, 23567.

11. Herbig, A. F.; Helmann, J. D. Mol. Microbiol. 2001, 41, 849.

12. Jacquamet, L.; Traore, D. A.; Ferrer, J. L.; Proux, O.; Testemale, D.; Hazemann, J. L.; Nazarenko, E.; El Ghazouani, A.; CauxThang, C.; Duarte, V.; Latour, J. M. Mol. Microbiol. 2009, 73, 20.

13. Lee, J. W.; Helmann, J. D. Nature 2006, 440, 363.

14. Lee, J. W.; Helmann, J. D. Biometals 2007, 20, 485.

15. Traore, D. A.; El Ghazouani, A.; Ilango, S.; Dupuy, J.; Jacquamet, L.; Ferrer, J. L.; Caux-Thang, C.; Duarte, V.; Latour, J. M. Mol. Microbiol. 2006, 61, 1211.

16. Traore, D. A.; El Ghazouani, A.; Jacquamet, L.; Borel, F.; Ferrer, J. L.; Lascoux, D.; Ravanat, J. L.; Jaquinod, M.; Blondin, G.; CauxThang, C.; Duarte, V.; Latour, J. M. Nat. Chem. Biol. 2009, 5, 53. 